\title{
MAGNETIC FIELD GENERATION BY BIERMANN BATTERY AND WEIBEL INSTABILITY IN LABORATORY SHOCK WAVES
}

\author{
G. Gregori ${ }^{1}$, F. Miniati ${ }^{2}$, B. Reville ${ }^{1}$ and R.P. Drake ${ }^{3}$
}

\begin{abstract}
Magnetic field generation in the Universe is still an open problem. Possible mechanisms involve the Weibel instability, due to anisotropic phase-space distributions, as well as the Biermann battery, due to misaligned density and temperature gradients. These mechanisms can be reproduced in scaled laboratory experiments. In this contribution we estimate the relative importance of these two processes and explore the laser-energy requirements for producing Weibel dominated shocks.
\end{abstract}

\section{Introduction}

With the advent of high-power laser systems in the past two decades, a new field of research has opened where, using simple scaling relations (Ryutov et al. 1999, 2000), astrophysical environments can effectively be reproduced in the laboratory (Remington et al. 1999,2006). In our previous work, we have demonstrated such capability and showed experimentally the generation of magnetic seeds by the Biermann effect at protogalactic shocks (Gregori et al. 2012). Those results have provided, for the first time, an experimental test of baroclinic models of magnetic field generation at shocks, and validating earlier numerical simulations (Kulsrud et al. 1997).

The Biermann battery mechanism is expected to produce tiny magnetic seed at cosmological shocks and, in order to explain present day values, significant amplification due to dynamo or turbulence must have occurred (Ryu et al. 2008). On the other hand, the Weibel instability (Weibel 1959), driven by the anisotropy of the particle velocity distribution, while playing an important role in mediating collision-less shocks, naturally generates $\mathrm{nG}$ magnetic fields (Schlickeiser \&

\footnotetext{
1 Department of Physics, University of Oxford, Parks Road, Oxford OX1 3PU, UK

2 Department of Physics, ETH Zürich, Wolfgang-Pauli-Strasse 27, 8093 Zürich, Switzerland

3 Department of Atmospheric, Oceanic, Space Science, University of Michigan, 2455 Hayward St, Ann Arbor, MI 48103, USA
} 
Shukla 2003; Medvedev 2007), at cosmic shocks. The fields, however, are characterized by microscopic scales, which makes them susceptible to rapid resistive dissipation. While survival of the fields against reconnection processes or other plasma instabilities need to be confirmed, Particle-in-cell (PIC) simulations are characterized by limited spatial and temporal scales (Medvedev 2007). In this respect, a direct laboratory experiment can provide very useful and complementary insight into whether or not coherent magnetic structures can emerge on macroscopic scales out of the initial microscopic ones in the turbulent postshock flow within appropriate timescales. In this paper, we will describe the laser requirements for the generation of Weibel mediated shocks based on the experimental platform described by Gregori et al. (2012).

\section{Magnetic field generation at shocks}

Given that laboratory and astrophysical shocks are never fully radially symmetric due to asymmetries in the initial drive and/or the presence of inhomogeneities in the ambient medium, there is always the possibility of a magnetic field generated through vorticity by Biermann's battery (Kulsrud et al. 1997; Hayes 1957)

$$
B_{\text {vort }}=\frac{m_{i} \omega}{e} \approx \frac{(\xi-1)^{2}}{\xi} \frac{m_{i}}{e(1+Z)}\left|\frac{\partial \mathbf{v}}{\partial S}\right|,
$$

where $\omega$ is the vorticity, $m_{i}$ is the ion mass, $e$ the electron charge, $Z$ is the ionization state, $\xi$ is the shock compression ratio ( $\sim 3-4$ for strong shocks), and $\partial \mathbf{v} / \partial S \sim \kappa v / r$ is the tangential gradient of the shock velocity, where $\mathbf{v}$ is the flow velocity and $\kappa$ is the asymmetry coefficient.

In the case of Weibel instability, PIC simulations have indicated that saturation of the magnetic field is reached when the ram pressure of the electron flow ahead of the shock is close to equipartition with the magnetic pressure (Medvedev 2007), that is,

$$
\frac{B_{w e i b e l}^{2}}{2 \mu_{0}} \sim \frac{1}{2} m_{e} n_{e} v^{2},
$$

where $m_{e}$ is the electron mass and $n_{e}$ is the electron density. It follows that magnetic fields produced by the Weibel instability will dominate over the Biermann effect (i.e., $B_{\text {weibel }}>B_{\text {vort }}$ ) when

$$
n_{e} r^{2}>\kappa^{2} \frac{(\xi-1)^{4}}{\xi^{2}} \frac{m_{p}^{2}}{\mu_{0} e^{2} m_{e}} \frac{A^{2}}{(1+Z)^{2}}
$$

where we have taken $m_{i}=A m_{p}$ (with $m_{p}$ is the proton mass). A further insight in the process may be obtained by realizing that in the Sedov-Taylor (energy conserving) phase, the shock wave must obey a relation of the type

$$
\frac{4 \pi}{3} r^{3} n_{i} m_{i} v^{2}=\eta E,
$$


where $E$ is energy deposited in the bast wave by an intense laser, $n_{i}=n_{e} / Z$ is the ion density, and $\eta$ is an efficiency factor. Hence, Weibel dominates when

$$
\frac{E}{v^{2} r}>\frac{4 \pi}{3} \frac{\kappa^{2}}{\eta} \frac{(\xi-1)^{4}}{\xi^{2}} \frac{m_{p}^{3}}{\mu_{0} e^{2} m_{e}} \frac{A^{3}}{Z(1+Z)^{2}} .
$$

The shock will continue to expand until radiative losses become important. Let's consider now the case of a pressure driven radiative shell (Ostriker \& McKee 1988). In the thin-shell limit we may approximate

$$
\frac{d E}{d t} \sim \frac{\eta E}{t}=4 \pi r^{2} \frac{n_{i} m_{i} v^{3}}{2}
$$

where $t$ stands for time. Combining 2.5 and 2.6 we obtain

$$
\frac{E^{2}}{t v^{5} r^{3}}>\frac{8 \pi^{2}}{3} \frac{\kappa^{2}}{\eta^{2}} \frac{(\xi-1)^{4}}{\xi^{2}} \frac{m_{p}^{4}}{\mu_{0} e^{2} m_{e}} \frac{A^{4}}{Z^{2}(1+Z)^{2}} n_{e}
$$

In addition, for the Weibel instability to actually develop, we need to ensure that the viscous dissipation time, $t_{\nu} \simeq \ell^{2} / \nu$, is much longer than the growth time of the instability, $t_{B} \simeq K \ell / v$ (Medvedev 2007). Here, $\ell \sim c / \omega_{p}$ is the spatial scale length, $\nu$ is the kinematic viscosity and $\omega_{p}$ is the ion plasma frequency, and $c$ is the speed of light. The coefficient $K>1$ indicates that the instability requires a certain number of ion skin depths in order to fully develop. From simulations $K \simeq 100-300$ (Kato \& Takabe 2008; Ross et al. 2012; Drake \& Gregori 2012). The condition $t_{\nu}>t_{B}$ can be rewritten as

$$
v>K \frac{\nu}{\ell}
$$

An identical relation can also be derived if, instead, we consider resistive diffusion of the magnetic field. In this case,

$$
v>K \frac{D_{M}}{\ell}
$$

where $D_{M}$ the magnetic diffusivity. This effectively ensures that the flux-freezing condition is satisfied. Moreover, using the approximation $v \sim \frac{3}{5} r / t$ (Draine \& McKee 1993), we obtain

$$
\frac{E}{r}>\frac{2^{3 / 2} \pi}{\sqrt{5}} \frac{\kappa K^{2}}{\eta} \frac{(\xi-1)^{2}}{\xi}\left(\frac{\epsilon_{0} c^{2} m_{p}^{5}}{\mu_{0} e^{4} m_{e}}\right)^{1 / 2}\left[\frac{A^{5}}{Z^{3}(1+Z)^{2}}\right]^{1 / 2} \frac{\nu D_{M}}{\ell^{3}} .
$$

We notice that there is some uncertainty in the previous estimates as the magnetic field can coalesce on spatial scales greater than $\ell$ even before saturation is reached.

\section{Application to laboratory experiments}

Let's now apply the result of Equation (2.10) to a case of a fully ionized hydrogen plasma $(A=1, Z=1)$, and under strong shock conditions $(\xi=4)$. While the coefficients $\kappa$ and $\eta$ depend on the details of the problem, some approximation based 
on experimental laboratory results can be performed. We should expect $\kappa \sim 0.1$ (Gregori et al. 2012) and $\eta \sim 0.01$. Taking $n_{e} \sim 10^{16} \mathrm{~cm}^{-3}$ and $T=10 \mathrm{eV}$, we get $\ell \simeq 0.2 \mathrm{~cm}, \nu \simeq 8.9 \times 10^{4} \mathrm{~cm}^{2} / \mathrm{s}$ and $D_{M} \simeq 4.7 \times 10^{5} \mathrm{~cm}^{2} / \mathrm{s}$. The condition expressed by Equation (2.10) (with $K=100$ ) now becomes

$$
E / r>0.6 \mathrm{MJ} / \mathrm{cm},
$$

which clearly indicates that only at the National Ignition Facility (NIF) generation of Weibel mediated collision-less shocks is feasible at $r \sim$ a few $\mathrm{cm}$ from the center of the blast wave. On NIF, we expect that a few MJ of laser energy will be available to drive the shock wave, hence, Weibel instability to significantly dominate. Experiments aimed at addressing these issues are currently under planning on NIF and on kJ laser facilities (Park et al. 2012).

The research leading to these results has received funding from the European Research Council under the European Community's Seventh Framework Programme (FP7/2007-2013) / ERC grant agreements No. 247039 and 256973. Partial support from the the Engineering and Physical Sciences Research Council of the United Kingdom (Grant No. EP/G007187/1) is also acknowledged.

\section{References}

Draine, B., \& McKee, C., 1993, Annual Rev. Astron. Astrophys., 31, 373

Drake, R.P., \& Gregori, G., 2012, ApJ, in press

Gregori, G., et al., 2012, Nature, 481, 480

Hayes, W.D., 1957, J. Fluid Mech., 2, 595

Kato, T.N., \& Takabe H., 2008, ApJ, 681, L93

Kulsrud, et al., 1997, ApJ, 480, 481

Medvedev, M.V., 2007, Ap\&SS, 307, 245

Ostriker, J.P., \& McKee, C.F., 1988, Rev. Mod. Phys., 80, 1

Park, H.-S., et al., 2012, High Energy Density Phys., 8, 38

Remington, B.A., et al., 1999, Science, 284, 1488

Remington, B.A., et al., 2006, Rev. Mod. Phys., 78, 755

Ross, J.S., et al., 2012, Phys. Plasmas, in press

Ryu, D., et al., 2008, Science, 320, 909

Ryutov, D.D., et al., 1999, ApJ, 518, 821

Ryutov, D.D., et al., 2000, ApJ, 127, S465

Schlickeiser, R., \& Shukla, P.K., 2003, ApJ, 599, L57

Weibel, E., 1959, Phys. Rev. Lett., 2, 83 